\title{
Influenza Vaccination Rates Among Homeless Adults With Mental Illness in Toronto
}

\author{
Samantha Young, Naheed Dosani, Adam Whisler, and \\ Stephen Hwang
}

Version Post-Print/Accepted Manuscript

Citation Young S., Dosani N., Whisler A., Hwang S. Influenza Vaccination Rates (published version) Among Homeless Adults With Mental Illness in Toronto. J Prim Care Community Health. 2015 Jul;6(3):211-4. doi:

10.1177/2150131914558881.

Publisher's Statement The final publication is available at SAGE Publications via http://dx.doi.org/10.1177/2150131914558881.

How to cite TSpace items

Always cite the published version, so the author(s) will receive recognition through services that track citation counts, e.g. Scopus. If you need to cite the page number of the TSpace version (original manuscript or accepted manuscript) because you cannot access the published version, then cite the TSpace version in addition to the published version using the permanent URI (handle) found on the record page. 


\title{
Influenza Vaccination Rates Among Homeless Adults With Mental IIIness in Toronto
}

Journal of Primary Care \& Community Health 2015, Vol. 6(3) 211-214

(C) The Author(s) 2014

Reprints and permissions:

sagepub.com/journalsPermissions.nav DOI: $10.1177 / 215013191455888$ I

jpc.sagepub.com

(SAGE

\author{
Samantha Young', Naheed Dosani ${ }^{2,3,4}$, Adam Whisler ${ }^{2}$, \\ and Stephen Hwang ${ }^{2,5}$
}

\begin{abstract}
Objective: To determine the incidence of influenza vaccination among homeless individuals with mental illness in Toronto. Methods: A retrospective chart review was carried out using a random sample of homeless individuals with mental illness who were participants of the At Home/Chez Soi Study. Primary care charts were obtained and reviewed for the incidence influenza vaccination within a I-year period. Results: Of the 75 participants for whom charts were reviewed, 5 (6.7\%) had documentation indicating receipt of the influenza vaccination in the year prior to study recruitment. Conclusion: This study raises awareness of the low incidence of homeless adults receiving the influenza vaccination in Toronto. The data are concerning given the high risk of morbidity from communicable respiratory illnesses in this group. Further research into the causes of low influenza vaccination rates among homeless Canadians is needed to develop strategies for increased delivery of the vaccination.
\end{abstract}

\section{Keywords}

primary care, prevention, influenza vaccination, homeless, mental illness

\section{Introduction}

Influenza remains a leading cause of vaccine-preventable hospitalizations and death. ${ }^{1,2}$ Current Canadian guidelines recommend universal influenza vaccination, but specifically note the importance of vaccinating certain high-risk groups, including those older than 65 years and those with chronic lung disease. Despite public health campaigns to encourage influenza vaccination, adherence rates remain low. In 2012, only $28.9 \%$ of Canadians and $31.1 \%$ of Ontarians received the flu shot. ${ }^{3}$

Homeless individuals suffer from a disproportionately high burden of respiratory illness and are likely to benefit from influenza vaccination. ${ }^{4}$ Because of high rates of smoking, chronic lung disease, poor nutrition, and crowded living conditions, homeless individuals are at increased risk for communicable respiratory diseases, including influenza. ${ }^{4-7}$ While influenza vaccination rates are known to be particularly low among marginalized urban populations, very little information is available on the rate of influenza vaccination in homeless populations in Canada. ${ }^{8,9}$ The objective of this study was to determine the rate of documented influenza vaccination in a cohort of homeless adults with mental illness in Toronto. There are more than 5000 homeless people in Toronto on any given night, with an estimated $38 \%$ experiencing current mental health problems. ${ }^{10,11}$ This study was conducted in a subsample of participants in the At Home/Chez Soi Study, a multicity randomized controlled trial investigating the effectiveness of a Housing First intervention for people who are homeless and have mental illness.

\section{Methods}

A total of 575 individuals who were homeless and had mental illness were enrolled at the Toronto site of the At Home/ Chez Soi Study from October 2009 to July 2011. Details of the research protocol have been previously published. ${ }^{12} \mathrm{~A}$ random sample of 200 study participants was selected for chart review. The research team sought to review primary care medical records for these 200 participants from the

\footnotetext{
'University of British Columbia, Vancouver, British Columbia, Canada

${ }^{2}$ St Michael's Hospital, Toronto, Ontario, Canada

${ }^{3}$ Inner City Health Associates, Toronto, Ontario, Canada

${ }^{4}$ McMaster University, Hamilton, Ontario, Canada

${ }^{5}$ University of Toronto, Toronto, Ontario, Canada

Corresponding Author:

Samantha Young, Centre for Research on Inner City Health, St. Michael's Hospital, 30 Bond Street, Toronto, Ontario, Canada M5B IW8.

Email: youngsamanthav@gmail.com
} 
Table I. Baseline Characteristics.

\begin{tabular}{lc}
\hline Characteristic & $\mathrm{n}(\%)$ \\
\hline Male sex & $49(65.3)$ \\
Age in years (mean \pm SD) & $39.1 \pm 12.2$ \\
Born in Canada & $43(57.3)$ \\
Have a provincial health card number & $70(93.3)$ \\
Current mental illness ${ }^{\mathrm{a}}$ & \\
Alcohol abuse/dependence & $28(37.3)$ \\
Drug abuse/dependence & $32(42.7)$ \\
Major depressive episode & $23(30.7)$ \\
Manic or hypomanic episode & $10(13.3)$ \\
Posttraumatic stress disorder & $18(24.0)$ \\
Mood disorder with psychotic features & $17(22.7)$ \\
Psychotic disorder & $30(40.0)$ \\
Panic disorder & $12(16.0)$ \\
\hline
\end{tabular}

a More than I mental illness diagnosis could be made for each participant.

year preceding enrollment in the study. Participants were asked to provide information on primary or ambulatory care received in the previous 18 months and consent for release of their medical records.

Charts were sought using physician contact information provided by participants or obtained from the College of Physicians and Surgeons of Ontario Web site. Physicians received a faxed request for information and the participant's signed consent for release of relevant medical records. Up to 5 follow-up phone calls were placed to request charts. The charts were obtained by various methods, including faxed or mailed copies, review of electronic medical records, and examination of charts at the physician's office. Data were abstracted using a standardized form to obtain information on diagnoses and preventive care interventions including influenza vaccination. To ensure reliability, a random $5 \%$ sample of charts were reviewed independently by 2 researchers.

\section{Results}

Of 200 participants, 3 did not consent to have their records released; 10 had not received any medical care or were unable to provide information on where they received care in the past 2 years; and 112 provided information for doctors who could not be located or did not provide primary care (usually a psychiatrist). In total, charts were obtained for 75 of 200 participants $(37.5 \%)$; some participants had medical charts obtained from multiple primary care sources. Baseline demographic data for these participants are shown Table 1.

Of 75 participants whose charts were reviewed, $5(6.7 \%)$ had documentation indicating receipt of the influenza vaccination in the year prior to study entry. The remaining 70 $(93.3 \%)$ had no documentation of receiving the influenza vaccination in their primary care chart.

\section{Discussion}

Vaccination represents one of the most successful public health interventions at the primary care level; the influenza vaccination is a cost-effective, proven method of decreasing respiratory illness complications and mortality ${ }^{2}$. Results of this study suggest that a very low proportion of homeless adults with mental illness are receiving this intervention. The rate of influenza vaccination $(6.7 \%)$ in this cohort of homeless adults with mental illness was less than one quarter of the vaccination rate among all Canadians $(28.9 \%){ }^{3}$

There are certain limitations to this study. The sample size is small, predominantly because of participants' inability to recall their primary care providers or a lack of contact with a primary care physician. This again highlights the limited access of homeless and mentally ill patients to primary care; in the general homeless population of Toronto, $43 \%$ report having a family physician, ${ }^{13}$ and the average annual number of primary care visits is 8 and 10 among homeless men and women, respectively. ${ }^{11}$ Second, this study includes only homeless adults with mental illness. The negative health consequences of homelessness tend to be more severe and access to primary care more limited in homeless individuals with mental illness when compared with those without mental illness. ${ }^{13}$ It is estimated that more than one third of homeless adults suffer from mental illness; thus, these individuals represent a significant proportion of the overall homeless population. ${ }^{14}$

Importantly, the chart review methodology may not capture influenza vaccinations received outside the primary care setting, such as at community "flu clinics." Toronto Public Health operates such clinics on approximately 14 days each year, but most clinics are not located in areas of the city where there are large numbers of homeless people, and in our experience very few homeless individuals report using these clinics. Finally, it is unknown how many patients were offered but declined a flu shot, rather than were not offered it at all. Nonetheless, the results of this study indicate that few of the homeless participants with mental illness successfully received an influenza vaccination from their primary care provider.

\section{Conclusions}

The extremely low proportion of homeless individuals in this study who received the influenza vaccination from their primary care provider is concerning given the high risk for morbidity in this group. Our results indicate a rate of vaccination much lower than several other studies done in US homeless populations that observed vaccination rates of between $21 \%$ and $35 \%{ }^{15-18}$ This study represents one of the few estimates of the rate of influenza vaccination among homeless adults in a Canadian population. Receiving the influenza vaccination is determined by many factors, 
including access to health care and personal views toward vaccination. ${ }^{8}$ It cannot be determined from this study what factors may have contributed to the low vaccination rate. Further research into the causes of low influenza vaccination rates among homeless Canadians is needed to generate strategies for increased vaccination delivery. Increased awareness among family physicians of the importance of influenza vaccination for homeless individuals is an important first step in primary prevention of influenza and its sequelae.

\section{Acknowledgments}

The authors thank the Toronto site research team, including research coordinators, research assistants, People With Lived Experience group members, service support providers, and participants for their contributions to the ongoing success of this study. The authors would like to acknowledge the national At Home/Chez Soi project team: Jayne Barker, PhD, and Cameron Keller, MHCC National Project Leads; Paula Goering, RN, PhD, Research Lead; approximately 40 investigators from across Canada and the United States; site coordinators in 5 cities; numerous service and housing providers; and persons with lived experience.

\section{Declaration of Conflicting Interests}

The author(s) declared no potential conflicts of interest with respect to the research, authorship, and/or publication of this article.

\section{Funding}

The author(s) disclosed receipt of the following financial support for the research, authorship, and/or publication of this article: This research has been made possible through a financial contribution from Health Canada and funded by the Mental Health Commission of Canada.

\section{References}

1. Parkins M, McNeil S, Laupland K. Routine immunization of adults in Canada: review of the epidemiology of vaccinepreventable diseases and current recommendations for primary prevention. Can J Infect Dis Med Microbiol. 2009;20: e81-e90.

2. Poland GA, Shefer AM, McCauley M, Webster PS, WhitleyWilliams PN, Peter G. Standards for adult immunization practices. Am J Prev Med. 2003;25:144-150.

3. Statistics Canada. Influenza Immunization, Less Than One Year Ago by Sex, by Province and Territory. Ottawa, Ontario: Statistics Canada; 2013. http://www.statcan.gc.ca/tablestableaux/sum-som/101/cst01/health102a-eng.htm. Accessed August 11, 2013.

4. Badiaga S, Richet H, Azas P, et al. Contribution of a shelterbased survey for screening respiratory diseases in the homeless. Eur J Public Health. 2009;19:157-160.

5. Hwang SW. Homelessness and health. CMAJ. 2001;164: 229-233.
6. Wright $\mathrm{MN}$, Tompkins $\mathrm{CN}$. How can health services effectively meet the health needs of homeless? Br J Gen Pract. 2006;56:286-293.

7. Raoult D, Foucault C, Brouqui P. Infections in the homeless. Lancet Infect Dis. 2001;1:77-84.

8. Bryant WK, Ompad DC, Sisco S, et al; Project VIVA Intervention Working Group. Determinants of influenza vaccination in hard-to-reach urban populations. Prev Med. 2006;43:60-70.

9. Task Force on Community Preventive Services. Recommendations regarding interventions to improve vaccination coverage in children, adolescents, and adults. Am J Prev Med. 2000;18:92-96.

10. City of Toronto. Street Needs Assessment Results 2013. Toronto, Ontario, Canada: Shelter, Support \& Housing Administration; 2013. http://www.toronto.ca/legdocs/mmis/2013/cd/bgrd/backgroundfile-61365.pdf. Accessed October 31, 2014.

11. Hwang SW, Chambers C, Chiu S, et al. A comprehensive assessment of health care utilization among homeless adults under a system of universal health insurance. Am J Public Health. 2013;103(suppl 2):S294-S301.

12. Goering PN, Streiner DL, Adair C, et al. The At Home/Chez Soi trial protocol: a pragmatic, multi-site, randomised controlled trial of a Housing First intervention for homeless individuals with mental illness in five Canadian cities. BMJ Open. 2011;1:e000323. doi:10.1136/bmjopen-2011-000323.

13. Khandor E, Mason K, Chambers C, Rossiter K, Cowan L, Hwang S. Access to primary health care among homeless adults in Toronto, Canada: results from the Street Health survey. Open Med. 2011;5:e94-e103.

14. Hwang SW, Wilkins R, Tjepkema M, O'Campo PJ, Dunn JR. Mortality among residents of shelters, rooming houses, and hotels in Canada: 11 year follow-up study. BMJ. 2009;339:b4036.

15. Ompad DC, Galea S, Blaney S, et al. Access to influenza vaccine in East Harlem and the Bronx during a national vaccine shortage. J Community Health. 2007; 32:195-202.

16. Coady M, Galea S, Blaney S, Ompad D, Sisco S, Vlahov D. Project VIVA: a multilevel community-based intervention to increase influenza vaccination rates among hardto-reach populations in New York City. Am J Public Health. 2008;98:1314-1321.

17. Bucher S, Brickner P, Vincent R. Influenza-like illness among homeless persons. Emerg Infect Diseases. 2006;12: 1162-1163.

18. Wiersma P, Epperson S, Terp S, et al. Episodic illness, chronic disease and health care use among homeless persons in Metropolitan Atlanta, Georgia, 2007. South Med J. 2010;103:18-24.

\section{Author Biographies}

Samantha Young, MD, is a resident in Internal Medicine at the University of British Columbia. She completed her medical degree at the University of Toronto.

Naheed Dosani, MD, CCFP, is a practicing physician in Family Medicine \& Palliative Care at St Michael's Hospital, 
Inner City Health Associates and William Osler Health System. $\mathrm{He}$ is an assistant clinical professor with the Faculty of Health Sciences: Department of Family Medicine at McMaster University.

Adam Whisler, BA, is a research analyst and Mental Health technician at the Centre for Addiction and Mental Health. He previously worked as a research coordinator at the Centre for
Research on Inner City Health at St. Michael's Hospital in Toronto.

Stephen Hwang, MD, MPH, FRCPC, is a practicing physician in general internal medicine and a research scientist at St. Michael's Hospital, Toronto. $\mathrm{He}$ is a professor of Medicine and holds the chair in Homelessness, Housing, and Health at St. Michael's Hospital and the University of Toronto. 